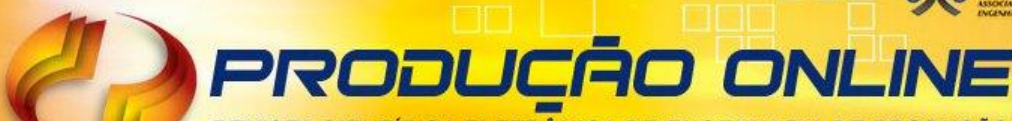 REVISTA CIENTIFICA ELETRÔNICA DE ENGENHARIA DE PRODUÇÃO ISSN 1676-1901
}

\section{APLICAÇÃO DA PROGRAMAÇÃO POR METAS E ANÁLISE POR ENVOLTÓRIA DE DADOS NO PROCESSO LICITATÓRIO DE EMBARCAÇÕES OFFSHORE DE UMA EMPRESA PETROLÍFERA}

\section{GOAL PROGRAMMING AND DATA ENVELOPMENT ANALYSIS (GPDEA) MODEL TO EVALUATE HIRING PROCESS FOR OFFSHORE VESSELS FOR AN OIL COMPANY}

\author{
Francisco Silva* E-mail: aneirson@yahoo.com.br \\ Fernando Augusto Silva Marins*E-mail: fmarins@feg.unesp.br \\ Rider Rodrigues Simões** E-mail: rider 5fly@yahoo.com.br \\ * Faculdade de Engenharia de Guaratinguetá (FEG-UNESP), Guaratinguetá, SP \\ ${ }^{* *}$ Fepi - Centro Universitário de Itajubá (FEPI-ITAJUBÁ), Porto Velho, MG
}

\begin{abstract}
Resumo: Este artigo tem por objetivo desenvolver e aplicar um modelo GPDEA-CCR da Programação por Metas e Análise por Envoltória de Dados (Goal Programming and Data Envelopment Analysis - GPDEA) para avaliar a eficiência do processo de contratação de embarcações offshore de uma empresa petrolífera. Este problema contempla vários requisitos técnicos, econômicos e legais que são necessários às operações logísticas offshore e já foi tratado por outros autores que adotaram um modelo tradicional da DEA-CCR. Foi abordado um problema real disponível na literatura envolvendo vinte embarcações candidatas à contratação pela empresa que foram consideradas como sendo as Unidades Tomadoras de Decisão (Decision Making Units DMUs) e, para cada uma delas, foram analisadas sete (7) variáveis, sendo duas (2) variáveis de entrada (inputs) e cinco (5) variáveis de saída (outputs). Os inputs avaliados para cada embarcação foram: o valor do custo diário de afretamento e o consumo de combustível. Os outputs avaliados para cada embarcação foram: a capacidade total de armazenamento de água potável; a capacidade total de armazenamento de óleo diesel; a capacidade total de armazenamento de carga de convés; a potência máxima contínua de propulsão, e a velocidade máxima a ser atingida durante a navegação. Os dois modelos, DEA-CCR e GPDEA-CCR, foram aplicados à mesma base de dados e compararam-se os seus desempenhos quanto à escolha da DMU (embarcação) mais interessante de ser contratada pela empresa. Observou-se que a aplicação da GPDEA permitiu uma melhor discriminação das alternativas analisadas, facilitando a tomada de decisão na contratação das embarcações offshore para uma empresa petrolífera. Foram testados também os conceitos da Fronteira Padrão, da Fronteira Invertida, da Fronteira Composta Normalizada e da Supereficiência para melhorar a discriminação das DMUs por ambos os modelos.
\end{abstract}

Palavras-chave: GPDEA. DEA. Eficiência. Processos licitatórios. Embarcações offshore. Empresa petrolífera.

Abstract: This article aims to develop and implement a Goal Programming and Data Envelopment Analysis (GPDEA) model to evaluate the efficiency of the hiring process for offshore vessels for an oil company. This situation features a number of technical, economic and legal requirements that are necessary for offshore logistics operations, and it has been treated by other authors by means a traditional DEA model. In a real problem addressed, the twenty vessels candidates for hiring by the company were considered to be the decision making units (DMUs ) and, for each, were analyzed seven (7) variables, two ( 2 ) variables input and five ( 5 ) output variables. The inputs evaluated for each vessel were: the value of the daily cost of chartering and fuel consumption. The outputs evaluated for each vessel were: total storage capacity of potable water, the total storage capacity of diesel, the total storage capacity of deck cargo, the maximum continuous power of propulsion, and the maximum speed to be reached during navigation. The two models, DEA and GPDEA, were applied to the same database and we compared their performance on the choice of DMU (vessel) more

Revista Produção Online, Florianópolis, SC, v.14, n. 4, p.1216-1234, out./dez. 2014. 
interesting to be hired by the company. It was observed that the application of GPDEA allowed a better discrimination of the alternatives analyzed, facilitating decision making in hiring offshore vessels for an oil company. The concepts of inverted frontier, composite and normalized frontier, and super efficiency were tested to improve the discrimination of DMUs for both models.

Keywords: GPDEA. DEA. Efficiency. Bidding process. Offshore vessels. Oil company.

\section{INTRODUÇÃO}

Empresas que atuam na área de exploração e produção de petróleo e gás natural têm necessidade de contratar, por meio de processos licitatórios, uma frota terceirizada de embarcações de apoio offshore para o suprimento as plataformas de petróleo. As embarcações de apoio marítimo são recursos críticos que têm tido uma demanda crescente no mercado brasileiro de produção de petróleo e gás offshore (MACIEL; MEZA, 2010). Normalmente o processo licitatório ocorre em duas etapas:

- Na primeira etapa são verificados os requisitos técnicos das propostas.

- Na segunda etapa, para as propostas técnicas classificadas, procede-se a análise das planilhas de preços.

Ocorre que, durante o período de vigência do contrato, após o processo licitatório, podem ser observados problemas técnico-operacionais que resultam em dispêndios adicionais para a empresa contratante (MACIEL et al., 2010), o que as têm motivado a utilizar métodos quantitativos que as auxiliem nestas importantes decisões envolvendo inúmeros aspectos econômicos, ambientais, técnicos e mesmo sociais.

Neste contexto, surge a oportunidade de se aplicar técnicas da Pesquisa Operacional (PO), como a técnica da Análise por Envoltória de Dados (Data Envelopment Analysis - DEA) e a Programação Por Metas (Goal Programming GP), que são métodos de tomada de decisão com múltiplos critérios (LORENTZIADIS, 2010 e MARINS et al., 2010). De fato, segundo Athanassopoulos (1995), Bal et al. (2010) e Silva et al. (2013a; 2013b) a DEA e a GP são importantes ferramentas da $\mathrm{PO}$, com diversas aplicações em diferentes áreas do conhecimento.

Sabe-se que a DEA é um procedimento bastante utilizado para analisar a eficiência de organizações (DMUs - Decision Making Units), tanto do setor público como privado, mas, em particular, quando o número de DMUs não é suficientemente maior que o total de variáveis (inputs e outputs) consideradas na modelagem, os modelos DEA tendem a identificar muitas DMUs como eficientes, ou seja, perdem seu poder de discriminação. Para enfrentar tal situação indesejada há algumas 
alternativas, como utilizar o conceito de supereficiência, modelos DEA com múltiplos critérios e também o método da eficiência cruzada, porém estas abordagens podem gerar problemas de inviabilidade e aumento da complexidade do modelo (BAL et al., 2010).

Outro problema identificado em aplicações de modelos DEA tem sido a geração de pesos irrealistas (unrealistic weight dispersion) para as variáveis, o que ocorre quando DMUs são classificadas com eficientes devido aos pesos dos inputs e outputs assumirem valores extremos, como zero. Neste caso, há técnicas de restrição aos pesos que tem sido propostas para evitar este tipo de problema, mas também têm sido relatados casos de soluções inviáveis para os pesos, além do fato que estes métodos acrescentam restrições ao modelo DEA tornando-o mais difícil de ser resolvido (BAL et al., 2010).

Neste artigo o objetivo geral foi realizar a avaliação da eficiência do processo licitatório de embarcações offshore para uma empresa do setor petrolífero, por intermédio de modelos de uma área conhecida por GPDEA. Pretende-se ilustrar as vantagens dos modelos GPDEA sobre os modelos básicos DEA.

Estes modelos da GPDEA, em geral, apresentam dispersões de pesos mais balanceadas do que os modelos DEA clássicos, além de diminuir o número de DMUs eficientes sem a necessidade de se impor restrições adicionais sobre os pesos. Estes modelos também apresentam a propriedade de serem invariantes com respeito às unidades das variáveis envolvidas (unit-invariance property).

Para a comparação dos dois modelos já citados, foram utilizados os dados do artigo de Maciel e Meza (2010), que aplicaram um modelo DEA tradicional para um problema da licitação de embarcações offshore para uma empresa petrolífera. Assim, foi realizada uma comparação dos resultados obtidos por estes autores e aqueles resultantes do modelo GPDEA, com respeito à capacidade de discriminação das DMUs eficientes. Adicionalmente, foram aplicados também os conceitos da fronteira invertida, e da supereficiência, que ajudam na discriminação das DMUs, para se verificar os impactos no poder de discriminação dos modelos.

Com respeito à classificação da pesquisa aqui relatada, segundo Bertrand e Fransoo (2002) e Miguel et al. (2012), ela pode ser classificada como sendo uma pesquisa aplicada, tendo objetivo empírico normativo, pois o modelo visa desenvolver políticas e estratégias que melhorem uma situação atual. A forma de abordar o problema é quantitativa, sendo o método de pesquisa a modelagem. 
Na seção 2 apresenta-se uma breve revisão sobre modelos de GP e DEA; na seção seguinte estão as etapas da pesquisa desenvolvida, incluindo a descrição do problema, a modelagem GPDEA e os resultados obtidos; na seção 4 estão as conclusões e recomendações para futuras pesquisas, seguidas das referências bibliográficas.

\section{PROGRAMAÇÃO POR METAS \& ANÁLISE POR ENVOLTÓRIA DE DADOS}

Charnes et al. (1978) e Banker et al. (1984) desenvolveram um método conhecido como Análise por Envoltória de Dados (Data Envelopment Analysis DEA) que permite, entre outras funcionalidades, comparar entidades similares, denominadas Unidades Tomadoras de Decisão (Decision Making Units - DMUs), que utilizam os mesmos recursos (inputs ou variáveis de entradas) e oferecem resultados (outputs ou variáveis de saídas) comparáveis. Deve ser observado que:

- As variáveis de entrada e de saída devem ser escolhidas de modo a representar o real interesse dos gestores.

- Devem existir dados numéricos positivos para as variáveis de entrada e de saída, sendo que se deve preferir um uso menor do número de entradas comparado ao de saídas.

Charnes et al. (1978) abordaram este tema pela primeira vez ao desenvolverem um modelo, formulado em (1) - (4), utilizando uma nova medida de eficiência $(E)$ na avaliação de programas públicos:

$$
\operatorname{Max} E=\sum_{r=1}^{s} u_{r} \cdot y_{r 0} / \sum_{i=1}^{m} v_{i} \cdot x_{i 0}
$$

S.a:

$$
\begin{aligned}
& \sum_{r=1}^{s} u_{r} y_{r j} / \sum_{i=1}^{m} v_{i} x_{i j} \leq 1, j=1,2, \ldots, n \\
& u_{r} \geq 0, \quad r=1,2, \ldots, s . \\
& v_{i} \geq 0, \quad i=1,2, \ldots, m .
\end{aligned}
$$

com $E$ sendo a eficiência relativa de $\mathrm{DMU}_{0}$, que é a $\mathrm{DMU}$ sob avaliação; $y_{r o}$ e $x_{i o}$ são, respectivamente, a r-ésima saída (output) e a i-ésima entrada (inputs) para $\mathrm{DMU}_{0}, u_{r}$ é o peso associado a r-ésima saída e $v_{i}$ é o peso associado a i-ésima 
entrada da $\mathrm{DMU}_{0}, y_{r j}$ é o valor da r-ésima saída para a j-ésima DMU e $x_{i j}$ é o valor da i-ésima entrada para a j-ésima DMU.

$\mathrm{Na}$ análise da solução do modelo (1) - (4), caso $E=1$, a $\mathrm{DMU}_{0}$ será considerada eficiente quando comparada às demais unidades consideradas no modelo; já se $E<1$, essa DMU será considerada ineficiente. Este modelo (1) - (4) não é linear, mas pode ser linearizado como formulado em (5) - (9), que é denominado modelo DEA-CCR ou modelo com Retornos Constantes de Escala (CHARNES et al.,1978):

$$
\max E=\sum_{r=1}^{s} u_{r} \cdot y_{r 0}
$$

s.a:

$$
\begin{aligned}
& \sum_{i=1}^{m} v_{i} x_{i o}=1 \\
& \sum_{r=1}^{s} u_{r} y_{r 0}-\sum_{i=1}^{m} v_{i} x_{i o} \leq 0, \quad j=1,2, \ldots, n \\
& u_{r} \geq 0, \quad r=1,2, \ldots, s . \\
& v_{i} \geq 0, \quad i=1,2, \ldots, m .
\end{aligned}
$$

Banker et al. (1984) relaxaram a suposição de retorno de escala constante do modelo DEA CCR, passando a admitir um retorno variável de escala e gerando o modelo conhecido como DEA BCC, formulado em (10) - (14):

$$
\max E=\sum_{r=1}^{s} u_{r} \cdot y_{r 0}+c_{0}
$$

s.a:

$$
\begin{aligned}
& \sum_{i=1}^{m} v_{i} x_{i o}=1 \\
& \sum_{r=1}^{s} u_{r} y_{r 0}-\sum_{i=1}^{m} v_{i} x_{i o}+c_{0} \leq 0, \quad j=1,2, \ldots ., n \\
& u_{r} \geq 0, \quad r=1,2, \ldots, s . \\
& v_{i} \geq 0, \quad i=1,2, \ldots, m . \quad c_{0} \text { Livre }
\end{aligned}
$$

A Figura 1 contempla um exemplo de avaliação de eficiência numa empresa com o único input sendo o número de funcionários e o único output o volume de vendas. Pela DEA é possível analisar a razão da produtividade, também conhecida 
como fronteira padrão de eficiência (BANKER et al.,1984). Exemplificando, na Figura 1 pode-se concluir que apenas a DMU B seria eficiente, pois se situa exatamente sobre esta fronteira. Nesta mesma figura, pelo conceito da fronteira de eficiência invertida da DEA, onde se invertem os papéis de inputs e outputs (COOPER et al.,2006) apenas a DMU F seria eficiente.

Figura 1- Exemplo de avaliação de eficiência

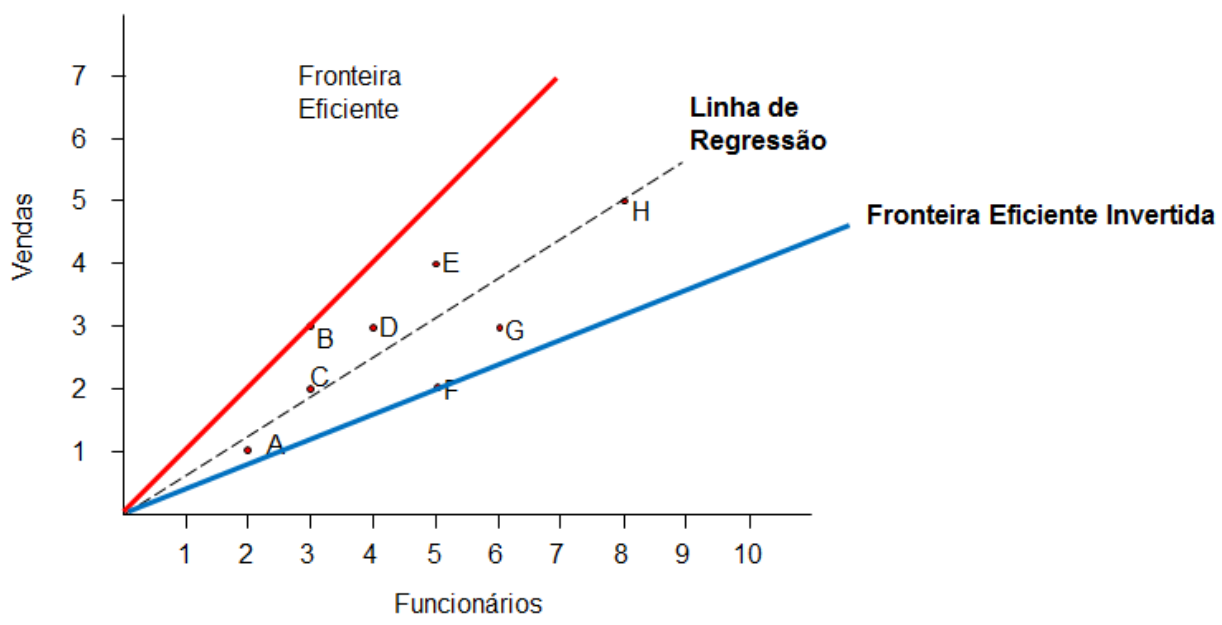

Fonte: Adaptado de Cooper et al. (2006)

$\mathrm{Na}$ aplicação da DEA, Banker et al. (1989) recomendam que o número de DMUs deve ser no mínimo igual ao produto do número de variáveis de entrada pelo número de variáveis de saída ou igual a 3 vezes o total de variáveis, adotando o critério que fornecer o maior número de DMUs. Esta condição é conhecida como a regra de ouro da DEA, e quando ela não é atendida os modelos tradicionais de DEA (CCR e BCC) tendem a não proporcionar uma boa discriminação das DMUs envolvidas, ou seja, várias DMUs podem ser classificadas como eficientes, dificultando o trabalho dos decisores. Este foi um aspecto detectado na aplicação do modelo DEA-CCR ao problema aqui abordado de licitação de embarcações offshore para uma empresa petrolífera.

Há métodos que contornam o problema desta baixa discriminação da DEA, como, por exemplo, incluir uma restrição aos pesos das entradas e das saídas. Ocorre que, em geral, estes procedimentos envolvem muita subjetividade por parte dos decisores o que pode gerar controvérsias e dificuldades.

Como alternativa ao enfoque somente pela DEA para o problema de licitação 
de embarcações offshore citado, procurou-se o apoio de modelos da Goal Programming (GP). A GP procura obter uma solução que atenda o maior número de objetivos, de interesse dos decisores, que estejam no modelo com múltiplos objetivos representativo do problema em questão (CHARNES; COOPER,1962, TAMIZ et al., 1998, SILVA et al., 2011, SILVA et al., 2012, SILVA et al., 2013a; SILVA et al., 2013b; JESUS et al., 2014).

Segundo Silva e Marins (2014) a GP possui muitos modelos, merecendo destaque a Lexicographic GP (LGP), também conhecida como Preemptive Goal Programming ou Programação por Metas com priorização; a Weighted GP (WGP), conhecida como GP Ponderada; e a MINMAX GP (MA). Estes são os três modelos mais utilizados nas aplicações disponíveis na literatura consultada (CABALLERO et al., 2009; YAGHOOBI et al., 2007; SILVA et al., 2011, SILVA et al., 2012, SILVA et al., 2013a, SILVA et al., 2013b, SILVA; MARINS, 2014).

No sentido de contornar o aspecto da baixa discriminação da DEA, quando a regra de ouro da DEA não é atendida, tem sido recomendada (COOPER et al. 2006, SILVA et al., 2013) a aplicação de modelos da GPDEA (BAL et al., 2010). O modelo GPDEA é derivado de um modelo multiobjetivo DEA, dado por (15) - (21), proposto por Li e Reeves (1999):

$$
\min _{0}\left(\text { ou } \max \sum_{r=1}^{s} \mathrm{u}_{r} \mathrm{y}_{r 0}\right)
$$

$\min M$

$$
\min \sum_{j=1}^{n} d_{j}
$$

S.a:

$$
\begin{aligned}
& \sum_{i=1}^{m} v_{i} x_{i o}=1 \\
& \sum_{r=1}^{s} u_{r} y_{r 0}-\sum_{i=1}^{m} v_{i} x_{i o}+d_{j}=0, \quad j=1,2, \ldots, n \\
& M-d_{j} \geq 0, j=1,2, \ldots, n \\
& u_{r} \geq 0, \quad r=1,2, \ldots, s . \\
& v_{i} \geq 0, \quad i=1,2, \ldots, m \text {. }
\end{aligned}
$$




$$
d_{j} \geq 0, \quad j=1,2, \ldots, n
$$

sendo $d_{0}$ a variável de desvio para a $\operatorname{DMU}_{0}$ (DMU que está em análise), com $d_{0} \in[0$, 1], $d_{j}$ é a variável de desvio para a $\operatorname{DMU}_{j} ; M=\max \left\{d_{j}\right\}$ é o valor máximo que as variáveis de desvio podem assumir e $M-d_{j} \geq 0$ na restrição (18) define o máximo desvio $M$ que não altera a região viável do problema.

$\mathrm{Na}$ análise da solução deste modelo (15) - (21), a $\mathrm{DMU}_{0}$ será eficiente se $d_{0}$ for igual a 0 e o valor da ineficiência é avaliado por (1 - $\left.d_{0}\right)$. Buscando incorporar os conceitos da GP neste último modelo para facilitar o trabalho dos decisores, Bal et al. (2010) associaram metas a cada uma das três funções objetivo em (15), e propuseram os modelos GPDEA-CCR e GPDEA-BCC, expressos respectivamente por (22) - (30) e (31) - (39), e descritos a seguir (SILVA et al., 2011, SILVA et al., 2012, SILVA; MARINS, 2014):

\section{Variáveis auxiliares}

$d_{1}^{-}, d_{1}^{+} \quad$ Variáveis de desvio indesejáveis para a meta que restringe a soma ponderada das entradas ao valor de 1 (100\%);

$d_{2}^{+} \quad$ Variável de desvio indesejável para a meta que restringe a soma ponderada das saídas a ser menor ou igual a 1 (100\%);

$d_{2}^{-} \quad$ Variável de desvio desejável para a meta que restringe a soma ponderada das saídas a ser menor ou igual a 1 (100\%);

$d_{3 j}^{-} \quad$ Variável de desvio indesejável, da DMU $j$, para a meta que tem $M$ como o desvio máximo;

$d_{3 j}^{+} \quad$ Variáveis de desvio desejável, da DMU $j$, para a meta que tem $M$ como o desvio máximo;

$d_{j} \quad$ Variável de desvio indesejável, da DMU j, com relação ao desvio máximo $M$;

M Variável de desvio máximo.

GPDEA-CCR

$$
\min \left(d_{1}^{-}+d_{1}^{+}+d_{2}^{-}+\sum_{j=1}^{n} d_{3 j}^{-}+\sum_{j=1}^{n} d_{j}\right)
$$


s.a:

$$
\begin{aligned}
& \sum_{i=1}^{m} v_{i} x_{i o}+d_{1}^{-}-d_{1}^{+}=1 \\
& \sum_{r=1}^{s} u_{r} y_{r 0}+d_{2}^{-}-d_{2}^{+}=1 \\
& \sum_{r=1}^{s} u_{r} y_{r 0}-\sum_{i=1}^{m} v_{i} x_{i o}+d_{j}=0, \quad j=1,2, \ldots, n \\
& M-d_{j}+d_{3 j}^{-}-d_{3 j}^{+}=0, \quad j=1,2, \ldots ., n \\
& u_{r} \geq 0, \quad r=1,2, \ldots, s . \\
& v_{i} \geq 0, \quad i=1,2, \ldots, m . \\
& d_{j} \geq 0, \quad j=1,2, \ldots, n . \\
& d_{i} \geq 0, \quad d_{3 j}^{-}, d_{3 j}^{+} \geq 0 .
\end{aligned}
$$

\section{GPDEA-BCC}

$$
\min \left(d_{1}^{-}+d_{1}^{+}+d_{2}^{-}+\sum_{j=1}^{n} d_{3 j}^{-}+\sum_{j=1}^{n} d_{j}\right)
$$

S.a:

$$
\begin{aligned}
& \sum_{i=1}^{m} v_{i} x_{i o}+d_{1}^{-}-d_{1}^{+}=1 \\
& \sum_{r=1}^{s} u_{r} y_{r 0}+c_{0}+d_{2}^{-}-d_{2}^{+}=1 \\
& \sum_{r=1}^{s} u_{r} y_{r 0}-\sum_{i=1}^{m} v_{i} x_{i o}+c_{0}+d_{j}=0, \quad j=1,2, \ldots, n \\
& M-d_{j}+d_{3 j}^{-}-d_{3 j}^{+}=0, j=1,2, \ldots, n \\
& u_{r} \geq 0, \quad r=1,2, \ldots, s . \\
& v_{i} \geq 0, \quad i=1,2, \ldots, m . \\
& d_{j} \geq 0, \quad j=1,2, \ldots, n . \\
& d_{i} \geq 0, \quad d_{3 j}^{-}, d_{3 j}^{+} \geq 0 .
\end{aligned}
$$

Neste trabalho propõe-se um Modelo GPDEA-CCR para avaliar a eficiência em processo licitatório de embarcações offshore para uma empresa petrolífera, tendo sido utilizados os dados do trabalho de Maciel e Meza (2010) para efeito de comparação. 


\section{ETAPAS DA PESQUISA}

As fases da pesquisa desenvolvida estão descritas na sequência:

Etapa (a) - Identificação do problema - o problema abordado, para a comparação dos modelos DEA-CCR e GPDEA-CCR, consiste em avaliar a eficiência de um processo licitatório de contratação de embarcações offshore para uma empresa petrolífera. As embarcações de apoio envolvidas são do tipo PSV (Platform Supply Vessel). Estas PSV se diferenciam entre si com relação aos seguintes fatores: o custo diário do afretamento e as condições operacionais.

Tabela 1- Dados para os modelos DEA e GPDEA. Fonte: Maciel e Meza (2010).

\begin{tabular}{cccccccc}
\hline & \multicolumn{3}{c}{ Inputs } & \multicolumn{5}{c}{ Outputs } \\
\cline { 2 - 8 } DMUs & $\begin{array}{c}\text { Custo } \\
{[\mathrm{US} \$ \text { /dia }]}\end{array}$ & $\begin{array}{c}\text { Combustível } \\
{[\text { ton/dia] }}\end{array}$ & $\begin{array}{c}\text { Água } \\
{\left[\mathrm{m}^{3}\right]}\end{array}$ & $\begin{array}{c}\text { Diesel } \\
{\left[\mathrm{m}^{3}\right]}\end{array}$ & $\begin{array}{c}\text { Carga } \\
{\left[\mathrm{m}^{2}\right]}\end{array}$ & $\begin{array}{c}\text { Potência } \\
{[\mathrm{BHP}]}\end{array}$ & $\begin{array}{c}\text { Velocidade } \\
{[\text { nós] }}\end{array}$ \\
\cline { 2 - 8 } PSV1 & 18.000 & 22,9 & 1.500 & 800 & 500 & 5.460 & 13 \\
PSV2 & 19.000 & 22 & 1.550 & 830 & 520 & 5.600 & 13,5 \\
PSV3 & 20.000 & 22,5 & 1.580 & 870 & 560 & 5.400 & 13,8 \\
PSV4 & 21.000 & 22,1 & 1.650 & 890 & 600 & 5.300 & 12,7 \\
PSV5 & 22.000 & 21,9 & 1.630 & 850 & 500 & 5.290 & 11,3 \\
PSV6 & 23.000 & 21,7 & 1.610 & 820 & 490 & 5.700 & 12,7 \\
PSV7 & 23.500 & 22,6 & 1.590 & 900 & 450 & 5.800 & 15,1 \\
PSV8 & 24.000 & 22,4 & 1.450 & 1.000 & 500 & 6.000 & 13,4 \\
PSV9 & 24.500 & 23,2 & 1.700 & 1.100 & 550 & 5.800 & 15,6 \\
PSV10 & 25.000 & 24,2 & 1.620 & 1.020 & 600 & 5.900 & 14,7 \\
PSV11 & 25.000 & 21,7 & 1.590 & 890 & 530 & 5.200 & 13,9 \\
PSV12 & 25.500 & 21 & 1.780 & 950 & 490 & 5.470 & 13,8 \\
PSV13 & 26.000 & 21,5 & 1.730 & 1.050 & 520 & 5.570 & 11,4 \\
PSV14 & 26.500 & 21,3 & 1.640 & 980 & 550 & 5.990 & 11,5 \\
PSV15 & 27.000 & 21,2 & 1.590 & 990 & 600 & 6.000 & 12,3 \\
PSV16 & 27.500 & 20,4 & 1.650 & 1.040 & 620 & 5.580 & 12,3 \\
PSV17 & 28.000 & 20,3 & 1.540 & 1.060 & 600 & 5.730 & 11,5 \\
PSV18 & 28.500 & 21,5 & 1.500 & 1.100 & 580 & 5.500 & 12 \\
PSV19 & 29.000 & 21,7 & 1.390 & 1.030 & 560 & 5.900 & 12,5 \\
PSV20 & 30.000 & 21,4 & 1.420 & 970 & 650 & 5.300 & 13 \\
\hline
\end{tabular}

Nota: BHP - HP Britânico, sendo 1 kW 1,341BHP e 1 nó 1,852 km/h.

Etapa (b) - Coleta dos dados - Os dados adotados foram extraídos de Maciel e Meza (2010), conforme Tabela 1, que utilizando dados reais avaliaram a eficiência do processo licitatório, já citado, por intermédio de um modelo DEA-CCR com 20 DMUs que correspondiam aos tipos de embarcações PSV propostos pelas vinte (20) 
empresas que participaram da licitação.

Estes autores consideraram no modelo DEA-CCR sete (7) variáveis sendo duas (2) variáveis de entrada (inputs) e cinco (5) variáveis de saída (outputs). Os inputs considerados para cada embarcação foram: o valor do custo diário ([US\$]) de afretamento e o consumo de combustível ([ton/dia]). Os outputs considerados para cada embarcação foram: a capacidade total de armazenamento de água potável $\left(\left[\mathrm{m}^{3}\right]\right)$; a capacidade total de armazenamento de óleo diesel $\left(\left[\mathrm{m}^{3}\right]\right)$; a capacidade total de armazenamento de carga de convés $\left(\left[\mathrm{m}^{2}\right]\right)$; a potência máxima contínua de propulsão ([BHP]), e a velocidade máxima a ser atingida durante a navegação ([nó]).

Observe-se que, pela regra de ouro já comentada, para aplicar o modelo DEA-CCR, neste caso seria recomendado que o número de DMUs fosse pelo menos vinte e um $(21=3.7)$.

Etapa (c) - Modelagem e Solução do Modelo - Neste artigo foi desenvolvido um Modelo GPDEA-CCR para o mesmo problema tratado por Maciel e Meza (2010). Este modelo foi implementado adotando-se o software The General Algebraic Modeling (GAMS) na versão 23.6 .5 e resolvido pelo solver CPLEX na versão 12.2.1.1.

Maciel e Meza (2010) trabalharam com modelo DEA-CCR tradicional e os resultados das eficiências das 20 DMUs que estes autores obtiveram estão na $2^{\text {a }}$. Coluna da Tabela 2 (Fronteira de Eficiência Padrão), ordenados do maior para o menor valor de eficiência. As demais colunas da Tabela 2 serão comentadas adiante. 
Tabela 2- Resultados de Eficiência para a Fronteira Padrão, Fronteira Invertida e Supereficiência com Modelo DEA-CCR (Maciel e Meza, 2010)

\begin{tabular}{|c|c|c|c|c|c|c|}
\hline \multirow[b]{2}{*}{ DMU } & \multicolumn{6}{|c|}{ Modelo DEA-CCR } \\
\hline & $\begin{array}{c}\text { Fronteira } \\
\text { de } \\
\text { Eficiência } \\
\text { Padrão } \\
\end{array}$ & $\begin{array}{l}\text { Fronteira } \\
\text { de } \\
\text { Eficiência } \\
\text { Invertida } \\
\end{array}$ & $\begin{array}{c}\text { Fronteira } \\
\text { Composta } \\
\text { Normalizada } \\
\text { (FCN) }\end{array}$ & $\begin{array}{l}\text { Ranking } \\
\text { (FCN) }\end{array}$ & $\begin{array}{c}\text { Supereficiência } \\
\text { (S) }\end{array}$ & $\begin{array}{c}\text { Ranking } \\
\text { (S) }\end{array}$ \\
\hline PSV1 & 1,0000 & 1,0000 & 0,9540 & 60 & 1,0291 & $7^{\circ}$ \\
\hline PSV2 & 1,0000 & 0,9532 & 0,9987 & $2^{\circ}$. & 1,0448 & $5^{\circ}$ \\
\hline PSV3 & 1,0000 & 0,9948 & 0,9589 & $5 \div$ & 1,0144 & $12^{0}$ \\
\hline PSV4 & 1,0000 & 0,9992 & 0,9540 & $6^{\circ}$. & 1,0595 & $3^{\circ}$ \\
\hline PSV7 & 1,0000 & 1,0000 & 0,9540 & $6^{\circ}$. & 1,0156 & $10^{\circ}$ \\
\hline PSV8 & 1,0000 & 1,0000 & 0,9540 & $6^{\circ}$. & 1,0141 & $13^{\circ}$ \\
\hline PSV9 & 1,0000 & 0,9708 & 0,9818 & $4 \stackrel{0}{ }$ & 1,0947 & $1 \stackrel{0}{2}$ \\
\hline PSV12 & 1,0000 & 1,0000 & 0,9540 & 60 & 1,0821 & $2^{\circ}$ \\
\hline PSV13 & 1,0000 & 1,0000 & 0,9540 & $6 \%$ & 1,0144 & $11^{\circ}$ \\
\hline PSV14 & 1,0000 & 1,0000 & 0,9540 & 60. & 1,0105 & $14^{\circ}$ \\
\hline PSV15 & 1,0000 & 0,9664 & 0,9860 & $3^{\circ}$. & 1,0188 & $9^{\circ}$ \\
\hline PSV16 & 1,0000 & 0,9518 & 1,0000 & $1 \%$ & 1,0539 & $4^{\circ}$ \\
\hline PSV17 & 1,0000 & 1,0000 & 0,9540 & 60. & 1,0296 & $6^{\circ}$ \\
\hline PSV20 & 1,0000 & 1,0000 & 0,9540 & 60. & 1,0047 & $15^{\circ}$ \\
\hline PSV18 & 0,9950 & 1,0000 & 0,9493 & $7^{\circ}$. & 1,0285 & $8^{\circ}$ \\
\hline PSV6 & 0,9889 & 1,0000 & 0,9435 & 80. & 0,9889 & $16^{\circ}$ \\
\hline PSV19 & 0,9755 & 1,0000 & 0,9307 & 90. & 0,9755 & $17^{\circ}$ \\
\hline PSV11 & 0,9740 & 1,0000 & 0,9293 & $10^{\circ}$. & 0,9740 & $18^{\circ}$ \\
\hline PSV5 & 0,9722 & 1,0000 & 0,9275 & $11^{\circ}$. & 0,9722 & $19^{\circ}$ \\
\hline PSV10 & 0,9679 & 0,9960 & 0,9272 & $12^{\circ}$. & 0,9679 & $20^{\circ}$ \\
\hline
\end{tabular}

Percebe-se, que o método aplicado não resultou numa boa discriminação com a fronteira padrão (2a. Coluna da Tabela 2), pois das vinte DMUs apenas seis DMUs (PSV5, PSV6, PSV10, PSV11, PSV18 e PSV19) não foram eficientes. Assim, pelo modelo DEA-CCR, no processo licitatório em questão ficariam "empatadas" 14 empresas concorrentes, o que dificultaria a decisão final com respeito a qual empresa contratar.

$\mathrm{Na}$ Tabela 3 têm-se os resultados com a aplicação do modelo GPDEA-CCR com os dados de Maciel e Meza (2010), e percebe-se que das 20 DMUs apenas seis DMUs (PSV2, PSV9, PSV12, PSV14, PSV15 e PSV16) foram eficientes pelo conceito de fronteira padrão (ver $2^{a}$. Coluna das Tabelas 2 e 3 ), o que já demonstra alguma vantagem do uso do modelo GPDEA-CCR. 
Tabela 3- Resultados obtidos com a aplicação do modelo de GPDEA-CCR

\begin{tabular}{|c|c|c|c|c|c|c|}
\hline \multirow[b]{2}{*}{ DMU } & \multicolumn{6}{|c|}{ Modelo GPDEA-CCR } \\
\hline & $\begin{array}{l}\text { Fronteira } \\
\text { Padrão }\end{array}$ & $\begin{array}{r}\text { Fronteira } \\
\text { Invertida }\end{array}$ & $\begin{array}{l}\text { Fronteira Composta } \\
\text { Normalizada (FCN) }\end{array}$ & $\begin{array}{l}\text { Ranking } \\
\text { FCN }\end{array}$ & Supereficiência (S) & $\begin{array}{l}\text { Ranking } \\
\mathrm{S}\end{array}$ \\
\hline PSV1 & 0,9519 & 1,0000 & 0,8720 & $14^{\circ}$ & 0,952 & $14^{\circ}$ \\
\hline PSV2 & 1,0000 & 0,9444 & 0,9670 & $5^{\circ}$ & 1,042 & $2^{\circ}$ \\
\hline PSV3 & 0,9667 & 0,9634 & 0,9190 & $11^{\circ}$ & 0,967 & $12^{o}$ \\
\hline PSV4 & 0,9649 & 0,9451 & 0,9340 & $8^{\circ}$ & 0,965 & $13^{\circ}$ \\
\hline PSV5 & 0,9387 & 1,0000 & 0,8600 & $16^{\circ}$ & 0,939 & $18^{\circ}$ \\
\hline PSV6 & 0,9726 & 0,9652 & 0,9230 & $10^{\circ}$ & 0,973 & $11^{\circ}$ \\
\hline PSV7 & 0,9755 & 0,9982 & 0,8950 & $13^{\circ}$ & 0,976 & $10^{\circ}$ \\
\hline PSV8 & 0,9861 & 0,9892 & 0,9130 & $12^{\circ}$ & 0,986 & $8^{\circ}$ \\
\hline PSV9 & 1,0000 & 0,9674 & 0,9460 & $7^{0}$ & 1,085 & $1^{\circ}$ \\
\hline PSV10 & 0,9443 & 0,9942 & 0,8700 & $15^{\circ}$ & 0,944 & $15^{\circ}$ \\
\hline PSV11 & 0,9248 & 1,0000 & 0,8470 & $19^{\circ}$ & 0,925 & $19^{\circ}$ \\
\hline PSV12 & 1,0000 & 0,9461 & 0,9650 & $6^{\circ}$ & 1,024 & $3^{\circ}$ \\
\hline PSV13 & 0,9849 & 0,9697 & 0,9300 & $9^{\circ}$ & 0,985 & $9^{0}$ \\
\hline PSV14 & 1,0000 & 0,9347 & 0,9760 & $4^{\circ}$ & 1,004 & $6^{\circ}$ \\
\hline PSV15 & 1,0000 & 0,9176 & 0,9920 & $2^{\circ}$ & 1,012 & $5^{\circ}$ \\
\hline PSV16 & 1,0000 & 0,9084 & 1,0000 & 10 & 1,022 & $4^{\circ}$ \\
\hline PSV17 & 0,9963 & 0,9245 & 0,9820 & $3^{\circ}$ & 0,996 & $7^{\circ}$ \\
\hline PSV18 & 0,9385 & 0,9997 & 0,8600 & $17^{\circ}$ & 0,944 & $16^{\circ}$ \\
\hline PSV19 & 0,9392 & 1,0000 & 0,8600 & $18^{\circ}$ & 0,939 & $17^{\circ}$ \\
\hline PSV20 & 0,8825 & 1,0000 & 0,8090 & $20^{\circ}$ & 0,883 & $20^{\circ}$ \\
\hline
\end{tabular}

Visando melhorar o poder de discriminação dos modelos DEA-CCR e GPDEA-CCR, pode-se usar a técnica da fronteira invertida, que consiste em considerar o que antes era input como sendo agora output e vice-versa, buscando, assim, realizar uma análise de eficiência do ponto de vista pessimista. Isto resultou nos valores da 3 a. Coluna da Tabela 2, que mostram que no modelo DEA-CCR das vinte DMUs, treze foram eficientes DMUs (PSV1, PSV7, PSV8, PSV12, PSV13, PSV14, PSV17, PSV20, PSV18, PSV6, PSV19, PSV11 e PSV5). Já com o modelo GPDEA-CCR, e a fronteira invertida, apenas cinco DMUs foram eficientes (PSV1, PSV5, PSV11, PSV19 e PSV20), conforme pode ser constatado na Tabela 3.

Continuando a comparação entre os dois modelos, outros dois conceitos que podem ser aplicados objetivando melhorar a discriminação das DMUs eficientes são: Fronteira Composta Normalizada (FCN) e Supereficiência (S). Segundo Cooper et al. (2006), a FCN pode ser obtida a partir da Fronteira Padrão e da Fronteira Invertida, fazendo-se:

- Fronteira Composta $=($ Fronteira Padrão - Fronteira Invertida +1$) / 2$.

- A Fronteira Composta Normalizada é obtida a partir da normalização dos valores encontrados para a Fronteira Composta.

Os resultados da FCN com o modelo DEA-CCR e com o modelo GPDEACCR indicaram que a DMU 16 (PSV16) deveria ser escolhida (ver 5ª. Coluna das 
Tabelas 2 e 3), mas discordaram no restante do ranking (ver Tabela 4).

Tabela 4- Comparações de rankings (quatro melhores) entre os modelos DEA-CCR e GPDEA-CCR

\begin{tabular}{ccccc}
\hline \multirow{2}{*}{ Ranking } & \multicolumn{2}{c}{ DEA-CCR } & \multicolumn{2}{c}{ GPDEA-CCR } \\
\cline { 2 - 5 } & $\begin{array}{c}\text { Fronteira } \\
\text { Composta } \\
\text { Normalizada } \\
\text { (FCN) }\end{array}$ & Supereficiência & $\begin{array}{c}\text { Fronteira Composta } \\
\text { Normalizada } \\
\text { (FCN) }\end{array}$ & Supereficiência \\
& PSV16 & PSV9 & PSV16 & PSV9 \\
$1^{\circ}$ & PSV2 & PSV12 & PSV15 & PSV2 \\
$2^{0}$ & PSV4 & PSV4 & PSV17 & PSV12 \\
$3^{0}$ & PSV9 & PSV16 & PSV14 & PSV16 \\
$4^{0}$ & & & & \\
\hline
\end{tabular}

Outra opção para melhorar a discriminação dos modelos é o uso da técnica de Supereficiência, conforme proposto por Andersen e Petersen (1993) e reafirmado por Xue e Harker (2002). Ela consiste na flexibilização da restrição que limita que a eficiência não pode ser maior que 1 (ou 100\%), como está estabelecido na restrição (7) do modelo DEA-CCR, anteriormente apresentado.

Desta forma, as DMUs que foram eficientes na análise inicial, com a utilização da técnica da supereficiência, poderão ter valores acima de 1. Com este artifício, pode-se realizar um ranqueamento adicional para estas DMUS eficientes, sem alterar os valores de ineficiência obtidos na análise inicial para as demais DMUs (que foram ineficientes). $O$ uso da Supereficiência nos modelos DEA-CCR e GPDEA-CCR resultou nos rankings que estão na $7^{\text {a }}$ Coluna das Tabelas 2 e 3 . Conforme resumido na Tabela 4, percebe-se que, com a Supereficiência, a DMU 9 é a que deveria ser selecionada em ambos os modelos (DEA-CCR e GPDEA-CCR). Contudo, há diferenças nas demais posições do ranking.

\section{CONCLUSÕES E RECOMENDAÇÕES PARA FUTURAS PESQUISAS}

Neste artigo foi realizada a avaliação da eficiência do processo licitatório de embarcações offshore para uma empresa do setor petrolífero, por intermédio de modelos de uma área conhecida por GPDEA. Foram avaliadas as vantagens dos modelos GPDEA sobre os modelos básicos DEA (CCR e BCC).

Para a comparação dos dois modelos já citados, foram utilizados os dados de Maciel e Meza (2010), que aplicaram um modelo DEA tradicional para um problema de licitação de embarcações offshore para uma empresa petrolífera. Assim, foi realizada uma comparação dos resultados obtidos por estes autores e aqueles 
resultantes do modelo GPDEA, com respeito à capacidade de discriminação das DMUs eficientes. Adicionalmente, foram aplicados também os conceitos da fronteira invertida, e da supereficiência, que ajudam na discriminação das DMUs, para se verificar os impactos no poder de discriminação dos modelos.

Percebe-se que, com a aplicação do DEA-CCR com a fronteira de eficiência padrão, das vinte DMUs apenas seis não foram eficientes, e com a aplicação do modelo GDPEA-CCR ocorreu o inverso, ou seja, das vinte DMUs apenas seis foram eficientes, demonstrando assim, o aumento da discriminação deste último modelo frente ao modelo DEA-CCR. Os demais testes com a fronteira composta normalizada e a supereficiência mostraram concordância parcial entre os rankings propostos pelos modelos analisados, sendo indicadas as mesmas DMUs para a 1‥ posição, respectivamente PSV16 e PSV9.

Como observações finais, sabe-se que a DEA é útil para se identificar quais os níveis (conhecidos como valores alvos) de outputs e inputs que uma DMU ineficiente deveria atingir para que se tornar eficiente. Assim um problema interessante é buscar uma relação de compromisso entre minimizar inputs ao mesmo tempo em que se tenta maximizar os outputs.

Em vista disto, como propostas para futuras pesquisas sugerem-se:

- a utilização de modelos DEA Aditivos.

- a adoção de abordagens mais recentes que utilizam conceitos da Teoria dos Jogos, como citado nos trabalhos de Wu et al. (2009), Du et al. (2011) e Santos et al. (2011) que podem também ajudar a lidar com esses dois objetivos conflitantes (maximizar outputs e minimizar inputs). Tais abordagens podem auxiliar em situações de processos de negociação para o contratante das embarcações que busca economizar em gastos com frete e consumo de combustível, ao mesmo tempo em que tenta obter o máximo de benefícios dos serviços oferecidos pelas embarcações contratadas.

\section{AGRADECIMENTOS}

Esta pesquisa teve apoio do CNPq - Conselho Nacional de Desenvolvimento Científico e Tecnológico (Processo No. 303362/2012-0 e Processo No. 470189/2012), da CAPES - Coordenação de Aperfeiçoamento de Pessoal de Nível 
Superior (Processo No. 024/2008) e da FAPESP - Fundação de Amparo à Pesquisa do Estado de São Paulo (Processo No. 2014/06374-2).

\section{REFERÊNCIAS}

AHERN, R. \& ANANDARAJAH, G. Railway projects prioritization for investment: Application of goal programming. Transport Policy, v.14, n.1. p. $70-80,2007$. http://dx.doi.org/10.1016/j.tranpol.2006.10.003

ANDERSEN, P. E PETERSEN, N. C. A procedure for ranking efficient units in data envelopment analysis. Management Science, v.39, p.1261-1264, 1993.

http://dx.doi.org/10.1287/mnsc.39.10.1261

ATHANASSOPOULOS, A. D. Goal Programming \& Data envelopment analysis (GoDEA) for target-based multi-level planning: Allocating central grants to the Greek local authorities. European Journal of Operational Research, v.87, n.3, p.535-550, 1995. http://dx.doi.org/10.1016/0377-2217(95)00228-6

BAL, H.; ÖRKCÜ, H. H.; ÇELEBIOĞLU, S. Improving the discrimination power and weights dispersion in the data envelopment analysis. Computers \& Industrial Engineering, v. 37, n.1, p.99-107, 2010.

BANKER, R. D.; CHARNES, A.; COOPER, W. W. Some models for estimating technical and scale inefficiencies in Data Envelopment Analysis. Management Science, v. 30, n.9, p.1078-1092, 1984. http://dx.doi.org/10.1287/mnsc.30.9.1078

BERTRAND, J. W. M.; FRANSOO, J. C. Operations management research methodologies using quantitative modeling. International Journal of Operations \& Production Management, v 22, n.2, p.241-264, 2002.

http://dx.doi.org/10.1108/01443570210414338

CHARNES, A.; COOPER, W. W. Programming with linear fractional functional. Naval Research Logistics Quarterly, v.9, n.3 e 4, p.181-185, 1962.

CHARNES, A.; COOPER, W. W. Management Model and Industrial Application of Linear Programming, v. 1. New York: Wiley, 1961.

CHARNES, A.; COOPER, W.W.;RHODES, E. Measuring the efficiency of decision making units. European Journal of Operational Research, v2, n.6, p.429-444, 1978.

COOPER, W. W.; SEIFORD, L. M.; TONE, K. Introduction to Data Envelopment Analysis and its uses: with DEA-Solver software and references. 1.ed. New York: Springer, 2006.

DE JESUS.; I.R.D.; GOMES, F.P.; MEZA, L.A. Avaliação da eficiência operacional das unidades de polícia pacificadora do estado do Rio de Janeiro. Produção online, v. 14, n.2, p. 448-464, 2014. http://dx.doi.org/10.14488/1676-1901.v14i2.1248 
DU, J., LIANG, L., CHEN, Y., COOK, W. D.; ZHU, J. A bargaining game model for measuring performance of two-stage network structures. European Journal of Operational Research, v.210, p.390-397, 2011.

http://dx.doi.org/10.1016/i.ejor.2010.08.025

JAMALNIA, A.; SOUKHAKIAN, M. A. A hybrid fuzzy goal programming approach with different goal priorities to aggregate production planning. Computers \& Industrial Engineering, v.56, n. 4, p.1474-1486, 2009.

http://dx.doi.org/10.1016/j.cie.2008.09.010

LI, X-B.; REEVES, G, R. A multiple criteria approach to data envelopment analysis. European Journal of Operational Research, vol. 115, n.3, p.507-517, 1999. http://dx.doi.org/10.1016/S0377-2217(98)00130-1

LORENTZIADIS, P. L. Post-objective determination of weights of the evaluation factors in public procurement tenders. European Journal of Operational Research, v.200, p. 261-267, 2010. http://dx.doi.org/10.1016/j.ejor.2008.12.013

MACIEL, G, S.; MEZA, L. A. Aplicação da Análise Envoltória de Dados nos processos licitatórios de contratação de embarcações offshore da Petrobras.

Simpósio Brasileiro de Pesquisa Operacional. SBPO, 2010.

MACIEL, G, S.; MEZA, L. A.; SILVEIRA, V. L. L. de. Utilização de DEA para análise de processos licitatórios estudo de caso do processo de contratação de embarcações de apoio marítimo offshore da Petrobras. Engevista, v.12, n.2, p.8192, 2010.

MARINS, F. A. S.; PEREIRA, M. S. do.; BELDERRAIN, M. C. N.; URBINA, L. M. S. Método de tomada de decisão com múltiplos critérios. 1a. ed, São Paulo:

Blucher Acadêmico, 2010.

MIGUEL, A. C. M.; FLEURY, A.; MELLO, C. H. P.; NAKANO, D. N.; TURRIONI, J. B.; HO, L. L.; MORABITO, R.; MARTINS, R. A.; PUREZA, V. Metodologia de pesquisa em engenharia de produção e gestão de operações. 1a. ed., Rio de Janeiro: Elsevier, 2010.

ROMERO, C. A general structure of achievement function for a goal programming model. European Journal of Operational Research, v. 153, n.3, p.675-686, 2004. http://dx.doi.org/10.1016/S0377-2217(02)00793-2

SANTOS, M. A. R., MARINS, F. A. S.; SALOMON, V. A. P., A utilização da função arbitragem de Nash nos modelos DEA CCR: uma abordagem na orientação combinada input/output sob a ótica da Teoria dos Jogos da Barganha. Simpósio Brasileiro de Pesquisa Operacional. SBPO, 2011.

TAMIZ, M.; JONES, D. F.; EL-DARZI, E. A review of Goal Programming and its applications. Annals of Operations Research, v. 58, n.1, p.39-53, 1995. http://dx.doi.org/10.1007/BF02032309

TAMIZ, M; JONES, D.; ROMERO, C. Goal programming for decision making: An overview of the current state-of-the-art. European Journal of Operational 
Research, v.111, n.3, p.569-581, 1998. http://dx.doi.org/10.1016/S0377$\underline{\text { 2217(97)00317-2 }}$

GENERAL ALGEBRAIC MODELING SYSTEM (GAMS).

http://gams.com/dd/docs/solvers/cplex.pdf. Acessado em 24/04/2010.

SILVA, A. F.; MARINS, F. A. S. Revisão da literatura sobre modelos de Programação por Metas determinística e sob incerteza. Produção, 2014 (aceito para publicação).

SILVA, A. F.; Ribeiro, I. M.; LOPES, P. R. \& MARINS, F. A. S. Uma Investigação sobre os Modelos de Programação de Metas Determinístico e Sob Incerteza:

Aplicação a Problemas de Planejamento Agregado em Usinas Sucroalcooleiras. In:

ENCONTRO NACIONAL DE ENGENHARIA DE PRODUÇÃO, 2011, Belo Horizonte.

SILVA, A. F.; Ribeiro, I. M; LOPES, P. R. \& MARINS, F. A. S. Método de Seleção de Variedades de Cana para o Plantio Utilizando a Programação de Metas \& Análise por Envoltória de Dados. In: ENCONTRO NACIONAL DE ENGENHARIA DE PRODUÇÃO, 2011.

SILVA, G. G. Avaliação da Eficiência de Unidades de Manufatura Baseada na Análise Envoltória de Dados. Dissertação (Mestrado em Engenharia de Produção) - Escola Federal de Engenharia de Itajubá, Universidade Federal de Itajubá, Itajubá, 2008.

SILVA, A. F.; MARINS, F. A. S.; MONTEVECHI, J. A. B. Multi-choice mixed integer goal Programming optimization in a sugar and ethanol milling company. Applied Mathematical Modelling, v. 37, n. 1, p. 6146-6261, 2013a.

http://dx.doi.org/10.1016/i.apm.2012.12.022

SILVA, A. F.; MARINS, F. A. S.; MONTEVECHI, J. A. B. Aplicação de programação por metas binária: mista em uma empresa do setor sucroenergético. Gestão \& Produção, v. 20, n. 2, p. 321-336, $2013 \mathrm{~b}$. http://dx.doi.org/10.1590/S0104-530X2013000200006

SILVA, A. F.; MARINS, F. A. S.; SANTOS, M. V. B. Programação por Metas e Análise Envoltória de Dados a Avaliação da Eficiência de Plantas Mundiais de Manufatura. Revista Eletrônica Pesquisa Operacional para o Desenvolvimento, v. 5, n. 2, p. 172-184, 2013.

URÍA, M. V. R.; CABALLERO, R.; RUIZ, F. \& ROMERO, C. Meta-goal programming. European Journal of Operational Research, v. 136, n.2, p.422-429, 2002. http://dx.doi.org/10.1016/S0377-2217(00)00332-5

YAGHOOBI M. A. \& TAMIZ, M. A method for solving fuzzy goal programming problems based on MINMAX approach. European Journal of Operational Research, v. 177, n.3, p.1580-1590, 2007. http://dx.doi.org/10.1016/i.ejor.2005.10.022

WU, J., LIANG, L., YANG, F.; YAN, H., Bargaining game model in the evaluation of decision making units. Expert Systems with Applications, v.36, p.4357-4362, 2009. 
Xue, M.; Harker, P.T. Ranking DMUs with Infeasible Super-Efficiency DEA Models. Management Science, v. 48, p. 05-710, 2002.

http://dx.doi.org/10.1016/i.eswa.2008.05.001

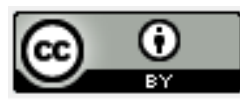

Artigo recebido em 09/07/2012 e aceito para publicação em 06/10/2014

DOI: http://dx.doi.org/ 10.14488/1676-1901.v14i4.1378 